\title{
Carcinoma urotelial plasmocitoide de vejiga urinaria. Estudio de 7 casos
}

\author{
Gaafar A, Garmendia M, de Miguel E*, Velasco V**, Ugalde A, Bilbao FJ, de Petris G***, \\ López JI**.
} Departamentos de Anatomía Patológica Hospital de Basurto. *Hospital de Galdakao. ${ }^{* *}$ Hospital de Cruces.
Universidad del País Vasco (EHU/UPV), Bizkaia. ${ }^{* * *}$ Mayo Clinic Scottsdale, AZ, USA.

Actas Urol Esp. 2008;32(8):806-810

\section{RESUMEN}

CARCINOMA UROTELIAL PLASMOCITOIDE DE VEJIGA URINARIA. ESTUDIO DE 7 CASOS

Objetivo: El carcinoma urotelial plasmocitoide es una variedad rara y agresiva de carcinoma vesical que histológicamente simula un plasmocitoma y que puede ser confundido con una neoplasia hemolinfoide que afecte secundariamente a la vejiga urinaria. Hasta la fecha solo se han descrito casos aislados y series cortas de esta variante neoplásica.

Pacientes y Método: Se han encontrado un total de 7 casos de carcinoma urotelial con fenotipo plasmocitoide total o parcial entre un total de 720 casos consecutivos de carcinoma urotelial de alto grado.

Resultados: El 0,97\% de los carcinomas vesicales uroteliales de alto grado analizados muestra morfología plasmocitoide. Todos los casos correspondieron a varones con edades comprendidas entre 58 y 75 años. Histológicamente, dos casos presentaban morfología plasmocitoide pura, mientras que en cinco de ellos el fenotipo plasmocitoide estaba combinado con áreas transicionales y/o glandulares convencionales. La inmunohistoquímica demostró en todos los casos el carácter epitelial de las áreas plasmocitoides. El comportamiento clínico fue agresivo en todos los casos, con metástasis a distancia en tres de ellos en el momento del diagnóstico y recurrencia precoz tras el tratamiento quimioterápico en cuatro.

Conclusiones: En nuestra experiencia, el carcinoma urotelial plasmocitoide de vejiga urinaria es muy poco frecuente, y puede presentarse de manera pura o asociado a áreas de carcinoma vesical convencional. Es conveniente reconocer esta variedad histológica debido a las implicaciones que conlleva en el pronóstico de los pacientes.

Palabras clave: Carcinoma urotelial. Fenotipo plasmocitoide. Alto grado. Pronóstico. Inmunohistoquímica.

\section{ABSTRACT}

PLASMACYTOID UROTHELIAL CARCINOMA OF THE URINARY BLADDER. A STUDY OF 7 CASES

Objective: Plasmacytoid urothelial carcinoma is a rare and aggressive variant of bladder cancer that mimics plasmacytoma histologically and that can be confused with hemolymphoid neoplasms secondarily affecting the urinary bladder. Only single cases and short series have been described so far.

Patients and Methods: Seven cases of plasmacytoid urothelial carcinoma have been found among 720 high grade urothelial carcinomas of the urinary bladder.

Results: In our series, $0.97 \%$ of high grade urothelial carcinomas of the urinary bladder show plasmacytoid phenotype. All the cases were smoking males between 58 and 75 years old. Histologically, two cases showed pure plasmacytoid features, while in the other five cases the plasmacytoid phenotype was mixed with conventional transitional cell or glandular histologies. By immunohistochemistry, all the plasmacytoid areas showed fair epithelial differentiation. The clinical behaviour was aggressive in all the cases, with distant metastases at diagnosis in three cases and early tumor recurrence after chemotherapy in four of them.

Conclusions: In our experience, the plasmacytoid urothelial carcinoma of the urinary bladder is a rare tumor that can also be detected in association with areas of conventional urothelial carcinoma. It is mandatory to recognize this histological subtype due to the clinical and prognostic implications of this diagnosis.

Keywords: Urothelial carcinoma. Plasmacytoid phenotype. High grade. Prognosis. Immunohistochemistry. 
$\mathrm{E}_{\mathrm{t}}^{\mathrm{l}}$ cáncer vesical es un tumor frecuente, que contabiliza aproximadamente el 3\% de las muertes por cáncer en los países desarrollados ${ }^{1}$. El carcinoma de células transicionales es, con mucho, la variedad histológica más frecuente, perteneciendo a la misma aproximadamente el 90\% de los casos. Además de esta variedad mayoritaria, se han descrito otras ${ }^{2,3}$, entre las que se encuentra la variedad plasmocitoide, que es muy poco frecuente ${ }^{4-13}$ y agresiva, y que puede confundirse histológicamente con neoplasias de estirpe hemolinfoide.

Este trabajo recopila 7 casos de carcinoma urotelial con fenotipo plasmocitoide y revisa la literatura concerniente a esta variedad histológica haciendo especial hincapié en los aspectos diagnósticos y pronósticos.

\section{PACIENTES Y MÉTODO}

Se han detectado 7 casos de carcinoma urotelial con fenotipo plasmocitoide en una revisión de 720 carcinomas uroteliales de alto grado. Todos los casos han sido tratados mediante resección transuretral y quimioterapia postquirúrgica mediante un régimen basado en cisplatino.

Los datos clínicos se han obtenido de las historias clínicas de los pacientes y el seguimiento se ha cerrado en Diciembre de 2007. Se han seleccionado en cada caso los bloques de parafina que contienen la diferenciación plasmocitoide, la cual se ha definido como "celularidad de morfología ovoidea con citoplasma eosinófilo con tendencia a la excentricidad y núcleos grandes, cromáticos, creciendo de forma difusa, sin patrón definido y de forma poco cohesiva, en ausencia de estructuras papilares glandulares, y de fenotipo transicional reconocible".

Las áreas seleccionadas en cada caso se han teñido con una batería de inmunohistoquímica que incluye: queratinas (AE1/AE3, CK7, CK20, Cam 5.2) antígeno de mem- brana epitelial (EMA), proteína S-100, CD45, desmina, antígeno carcinoembrionario (CEA) CA19.9, hormona gonadotropínica humana, fracción beta (bHCG) y Ki67.

\section{RESULTADOS}

Los datos clínicos aparecen resumidos en la Tabla 1. Todos los pacientes eran varones fumadores con edades comprendidas entre 58 y 75 años (media, 69). Todos los casos se presentaron clínicamente con hematuria. En el momento del diagnóstico tres casos mostraron metástasis a distancia, con carcinomatosis peritoneal en dos de ellos y masa en partes bandas del muslo izquierdo en otro. Los exámenes radiológicos revelaron engrosamiento difuso de la pared vesical, hecho indicativo de enfermedad músculo infiltrante, en todos los casos. Se detectó obstrucción ureteral e hidronefrosis en dos casos, siendo bilateral en uno de ellos.

El examen histológico (Tabla 2) demostró una histología mixta, con áreas de carcinoma de células transicionales papilar convencional de alto grado en cinco casos, uno de los cuales (caso 1), además, presentó áreas micropapilares, y otro de ellos (caso 5),

Tabla 1. Datos Clínicos

\begin{tabular}{cllll}
\hline Caso & Edad/Sexo & Presentación & Metastasis & Tto/Recurrencias \\
\hline 1 & $75 /$ Varón & Hematuria & Carcinomatosis peritoneal & Quimioterapia \\
2 & $70 /$ Varón & Hematuria & Muslo izquierdo & Quimioterapia \\
3 & $74 /$ Varón & Hematuria & No & Quimioterapia \\
4 & $73 /$ Varón & Hematuria & $\begin{array}{l}\text { Carcinomatosis peritoneal } \\
\text { Invasión pared intestinal }\end{array}$ & Quimioterapia \\
& & & No & Quimioterapia \\
5 & $65 /$ Varón & Hematuria & No & Cistectomía radical \\
6 & $58 /$ Varón & Hematuria & Guimioterapia \\
& & Hidronefrosis & & $\begin{array}{l}\text { Cistectomía radica } \\
\text { Quimioterapia }\end{array}$ \\
\hline
\end{tabular}

Tabla 2. Datos Histológicos

\begin{tabular}{cllcr}
\hline Caso & Histología & Diferenciaciones & Grado & pT \\
\hline 1 & Mixta & CCT + micropapilar + plasmocitoide & 3 & 2 \\
2 & Mixta & CCT + plasmocitoide & 3 & 2 \\
3 & Mixta & CCT + plasmocitoide & 3 & 3 \\
4 & Pura & Plasmocitoide & 3 & 4 \\
5 & Mixta & CCT + plasmocitoide + glandular + anillo de sello & 3 & 3 \\
6 & Pura & Pasmocitoide & 3 & 4 \\
7 & Mixta & CCT + plasmocitoide & 3 & 2 \\
\hline
\end{tabular}

CCT: Carcinoma de células transicionales 
áreas glandulares. Sólo dos de los casos de la serie presentaron histología plasmocitoide pura (casos 4 y 6). El componente plasmocitoide (Fig. 1) consistió en un crecimiento neoplásico poco cohesivo compuesto por células de núcleos atípicos, hipercromáticos, con abundantes mitosis, y citoplasmas eosinófilos, a veces mostrando imágenes de "anillo de sello". Los resultados del estudio inmunohistoquímico aparecen reflejados en la Tabla 3 y muestran el carácter inequívocamente epitelial de este componente neoplásico.

Cinco pacientes presentaron recurrencia de la enfermedad tras completar el tratamiento quimioterápico y tres de ellos fallecieron a consecuencia de la enfermedad en los primeros 2 años de seguimiento. En la actualidad, dos pacientes siguen el tratamiento post re-
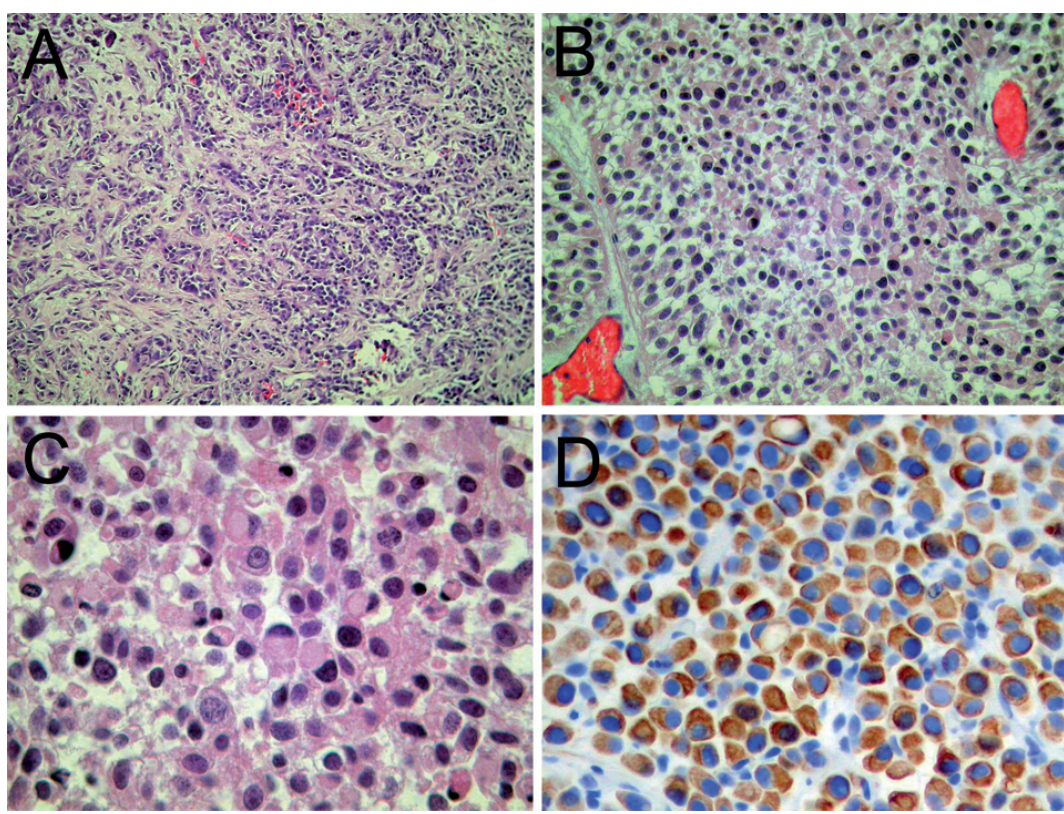

FIGURA 1. Histología característica en el carcinoma urotelial plasmacitoide: (A): Patrón de infiltración difuso, (B) Células malignas de hábito plasmoide, con núcleos excéntricos y citoplasmas eosinofilicos, (C) Céluls " en anillo de sello", (D) positividad intensa para queratina AE1/AE3. sección transuretral basado en el cis-

platino y se encuentran sin enfermedad aparente tras 4 y 8 meses de seguimiento, respectivamente.

\section{DISCUSIÓN}

Entre patólogos, es un hecho bien conocido que existe una amplia variedad de tumores que pueden mostrar una apariencia plasmocitoide en cortes convencionales, incluyendo entidades tan dispares como fibrosarcomas ${ }^{14}$, adenomas monomorfos de glándula salival ${ }^{15}$, o linfomas no hodgkinianos ${ }^{16}$ entre otros. La variedad plasmocitoide es muy poco frecuente en el carcinoma urotelial, con únicamente 30 casos previamente publicados hasta la fecha ${ }^{3}$. El diagnóstico suele dilatarse en el tiempo debido a la tardía aparición de la hematuria y a la induración de la mucosa en el examen cistoscópico ${ }^{13}$. Desde el punto de vista clínico es importante reconocer esta variedad histológica puesto que se asocia invariablemente a mal pronóstico.

Las primeras descripciones de esta variante histológica datan de 1991, cuando Zukerberg et al. ${ }^{5}$ y Sahin et al. ${ }^{4}$ publicaron casos aislados haciendo entonces hincapié en su similitud con el plasmocitoma. Desde entonces sólo se han descrito casos sueltos y pequeñas series de hasta 7 casos $^{4-13}$. En una serie reciente, Perez-Montiel et al. ${ }^{10}$ encuentran sólo un caso de carcinoma urotelial con fenotipo plasmocitoide parcial entre 108 carcinomas uroteliales de alto grado, lo cual confirma nuestra experiencia de una frecuencia por debajo del $1 \%$.

Tabla 3. Datos inmunohistoquímicos

\begin{tabular}{|c|c|c|c|c|c|c|c|c|c|c|c|}
\hline Caso & $\mathrm{AE} 1 / 3$ & EMA & CK7 & CK20 & CAM 5.2 & CEA & S-100 & CD45 & CA19.9 & Bhcg & Ki67* \\
\hline 1 & + & + & + & $+/-$ & + & - & - & - & - & - & $0 \%$ \\
\hline 2 & + & + & + & - & + & - & - & - & $+/-$ & - & $90 \%$ \\
\hline 3 & $+/-$ & + & + & - & - & - & - & - & - & - & $90 \%$ \\
\hline 4 & + & + & + & $+/-$ & + & + & - & - & + & - & $50 \%$ \\
\hline 5 & + & + & + & - & - & - & - & - & $+/-$ & - & $60 \%$ \\
\hline 6 & + & + & + & - & - & - & - & - & + & - & $70 \%$ \\
\hline 7 & + & + & + & - & - & - & - & - & - & - & $40 \%$ \\
\hline
\end{tabular}


Desde el punto de vista histológico, lo más frecuente es que el componente plasmocitoide se asocie a áreas de carcinoma de células transicionales convencional, habitualmente de alto grado e infiltrante. No obstante, Coyne y $\mathrm{Sim}^{11}$ publican un caso de carcinoma urotelial no invasor con componente plasmocitoide. Dentro de la rareza es aún mucho menos frecuente encontrar carcinomas uroteliales plasmocitoides puros, como ocurre en dos de los casos presentados en este trabajo. Obviamente, es en estos casos en los que el diagnóstico diferencial con neoplasias hemolinfoides es más complicado. Por ejemplo, el caso publicado por Sahin et al. ${ }^{4}$ debutó con lesiones metastásicas óseas múltiples mimetizando fielmente un mieloma. Sólo la cistoscopia realizada para el estudio de una hematuria intercurrente reveló la verdadera naturaleza del tumor ${ }^{4}$. Es en estos casos en los que la inmunohistoquímica es una herramienta diagnóstica crucial $^{17}$.

El diagnóstico diferencial de esta variedad histológica de cáncer vesical incluye principalmente a los plasmocitomas que afecten secundariamente a la vejiga urinaria, y en segundo término al carcinoma urotelial de tipo linfoepitelioma, el carcinoma de células pequeñas, el rabdomiosarcoma embrionario, y el melanoma ${ }^{3,12}$. Para tal fin, un panel compuesto por queratinas de amplio espectro, proteína S-100, desmina, EMA y CD45 es lo apropiado. El carácter epitelial de la neoplasia quedará refrendado por la positividad para AE1/AE3, Cam 5.2 y CK7 y EMA en el caso que nos ocupa ${ }^{11,13}$. La CK20 es negativa. El melanoma será positivo con la proteína S-100 y el rabdomiosarcoma con la desmina. Adicionalmente, las queratinas mostrarán un patrón de tinción citoplásmico "en punto" en el carcinoma de células pequeñas, y precisarán con nitidez el nivel de invasión del cáncer en la variedad de carcinoma vesical tipo linfoepitelioma, en el que el componente epitelial maligno se encuentra muchas veces oscurecido o ahogado en un magma de células linfoides no neoplásicas. En el caso de encontrar células "en anillo de sello" éstas pueden ser positivas con $\mathrm{CEA}^{13}$. El perfil inmunohistoquímico comentado hasta ahora debería ser suficiente en todos los casos.

Existen múltiples referencias que indican que el carcinoma urotelial plasmocitoide puede ser positivo frente a marcadores de diferenciación plasmoce- lular como el CD1387-9,13, lo cual lo hace equívoco y por lo tanto inservible en el diagnóstico diferencial. En el caso de utilizarlo y ser positivo, se precisará realizar, además, técnicas inmunohistoquímicas para cadenas ligeras kappa y lambda para resolver de manera fiable el dilema ${ }^{17}$. Se han descrito otros marcadores, pero son menos efectivos para el diagnóstico diferencial. Shimada et al. ${ }^{9}$, por ejemplo, encuentran elevación sérica de CA 19.9 y de bHCG, lo cual se refrenda posteriormente en el análisis inmunohistoquímico, ya que ambas sustancias se detectan fundamentalmente en las células de hábito plasmocitoide.

Colabufo et al. ${ }^{18}$ demuestra sobre-expresión de receptores sigma ${ }_{2}$ y de Ki67 en los carcinomas de células transicionales de alto grado convencionales. Sin que se sepa aún la razón concreta, la expresión de Ki67 en el carcinoma urotelial plasmocitoide y en el carcinoma epidermoide vesical puede ser baja o incluso nula y sin embargo es muy intensa en el tejido peritumoral ${ }^{18}$. Curiosamente, las áreas con baja expresión de Ki67 coinciden con las de baja expresión de receptores sigma ${ }_{2}$.

Cualquier carcinoma vesical diseminado tiene mal pronóstico independientemente de cual sea la variedad histológica ${ }^{17}$. La cistectomía radical debería ser de elección en casos de enfermedad no metastásica, aunque también se ha planteado la quimioterapia con preservación vesical como alternativa válida. Es difícil determinar cual es el tratamiento más efectivo en el carcinoma urotelial plasmocitoide debido a la rareza del tumor y a la falta de experiencia. En general, se trata de un tumor muy agresivo, con altas cifras de muerte relacionada con la enfermedad en los primeros dos años tras el diagnóstico.

\section{REFERENCIAS}

1. Jemal A, Siegel R, Ward E, Murray T, Xu J, Thun MJ. Cancer statistics, 2007. CA Cancer J Clin. 2007;57(1):43-66.

2. Lopez-Beltran A, Cheng L. Histologic variants of urothelial carcinoma: differential diagnosis and clinical implications. Hum Pathol. 2006;37(11):1371-1388.

3. Zhai QJ, Black J, Ayala AG, Ro JY. Histologic variants of infiltrating urothelial carcinoma. Arch Pathol Lab Med. 2007;131(8):1244-1256.

4. Sahin AA, Myhre M, Ro JY, Sneige N, Dekmezian RH, Ayala AG. Plasmacytoid transitional cell carcinoma. Report of a case with initial presentation mimicking multiple myeloma. Acta Cytol. 1991;35(3):277-280.

5. Zukerberg LR, Harris NL, Young RH. Carcinomas of the urinary bladder simulating malignant lymphoma. A report of five cases. Am J Surg Pathol. 1991;15(6):569-576. 
6. Zhang XM, Elhosseiny A, Melamed MR. Plasmacytoid urothelial carcinoma of the bladder: A case report and the first description of urinary cytology. Acta Cytol. 2002;46(2):412 416.

7. Soylu A, Aydin NE, Yilmaz U, Kutlu R, Gunes A. Urothelial carcinoma featuring lipid cell and plasmacytoid morphology with poor prognostic outcome. Urology. 2005;65(4):797.

8. Mitsogiannis IC, Ioannou MG, Sinani CD, Melekos MD. Plasmacytoid transitional cell carcinoma of the urinary bladder. Urology. 2005;66(1):194.

9. Shimada K, Nakamura M, Ishida E, Konishi N. Urothelial carcinoma with plasmacytoid variants producing both human chorionic gonadotropin and carbohydrate antigen 19-9. Urology. 2006;68(4):891.e7-10

10. Perez-Montiel D, Wakely PE, Hes O, Michal M, Suster S. Highgrade urothelial carcinoma of the renal pelvis: clinicopathologic study of 108 cases with emphasis on unusual morphologic variants. Mod Pathol. 2006;19(4):494-503.

11. Coyne JD, Sim E. Urothelial neoplasia with plasmacytoid morphology. Histopathology. 2006;48(2):200-201.

12. Kohno T, Kitamura M, Akai H, Takaha M, Kawahara K, Oka T. Plasmacytoid urothelial carcinoma of the bladder. Int $\mathrm{J}$ Urol. 2006; 13(4):485-486

13. Mai KT, Park PC, Yazdi HM, Saltel E, Erdogan S, Stinson WA, et al. Plasmacytoid urothelial carcinoma of the urinary bladder report of seven new cases. Eur Urol. 2006;50(5):11111114
14. Antonescu CR, Erlandson RA. Fibrosarcoma mimicking plasmacytoma or carcinoma: an ultrastructural study of 4 cases. Ultrastruct Pathol. 2001;25(1):31-37.

15. Franquemont DW, Mills SE. Plasmacytoid monomorphic adenoma of salivary glands. Absence of myogenous differentiation and comparison to spindle cell myoepithelioma. Am J Surg Pathol. 1993;17(2):146-153.

16. Pangalis GA, Nathwani BH, Rappaport H. Malignant lymphoma, well differentiated lymphocytic. Its relationship with chronic lymphocytic leukemia and macroglobulinemia of Waldenstrom. Cancer 1977;39(3):999-1010.

17. McKenney JK, Amin MB. The role of immunohistochemistry in the diagnosis of urinary bladder neoplasms. Semin Diagn Pathol. 2005;22(1):69-87.

18. Colabufo NA, Berardi F, Contino M, Ferorelli S, Niso M, Perrone $\mathrm{R}$,et al. Correlation between sigma2 receptor protein expression and histopathologic grade in human bladder cancer. Cancer Lett. 2006;237(1):83-88.

Correspondencia autor: Dr. J.I. López

Departamento de Anatomía Patológica. Hospital de Cruces

Plaza de Cruces, s/n - 48903 Baracaldo, Vizcaya

Tel.: 946006336

E-mail autor: joseignacio.lopez@ehu.es

Información artículo: Original - Cáncer de vejiga

Trabajo recibido: marzo 2008

Trabajo aceptado: mayo 2008 